\title{
Informalidad y segmentación del mercado laboral: el caso de la Argentina
}

\author{
Luis Beccaria y Fernando Groisman
}

RESUMEN

En este documento se evalúa la presencia de segmentación en el mercado laboral argentino. El análisis se centra en la comparación de los ingresos de los trabajadores formales e informales, mediante dos enfoques distintos sobre la definición de informalidad. Se comprueba la existencia de una prima formal por medio de la utilización de datos dinámicos y técnicas semiparamétricas. Se analizó el período comprendido entre 1996 y 2006 de todas las zonas urbanas encuestadas. Los resultados obtenidos respaldan la hipótesis de la segmentación del mercado laboral urbano de la Argentina, esto es, que trabajadores que cuentan con probabilidades similares para transitar de un sector a otro perciben ingresos diferentes. gmail.com

Fernando Groisman es Investigador del Consejo Nacional de Investigaciones Científicas y Técnicas (conicet)-Universidad de Buenos Aires (Uba), Argentina. groismanf@hotmail.com 


\section{I}

\section{Introducción}

La persistencia de altos niveles de informalidad en América Latina, incluso durante períodos de crecimiento económico, ha dado origen a numerosas investigaciones sobre el tema. Gran parte de ellas se han enfocado en probar la hipótesis de un mercado laboral dual o segmentado, principalmente mediante la evaluación empírica de las brechas de ingreso entre el trabajo formal y el informal ${ }^{1}$. En la mayoría de estos estudios se ha descubierto una prima positiva a la formalidad, resultado que suele producir acalorados debates acerca de sus consecuencias. Específicamente, en una línea argumental se vincula a estos resultados con la existencia de segmentación; sin embargo, hay diferencias en cuanto a las causas de ese fenómeno ${ }^{2}$.

Desde un determinado punto de vista, las brechas salariales constituyen una de las consecuencias de las limitaciones en materia de competencia, como los reglamentos laborales o los sindicatos. No obstante, desde otra perspectiva, en condiciones de baja demanda laboral, algunos trabajadores no tienen acceso a puestos del sector formal y se ven obligados a aceptar trabajos informales con menores ingresos. En este contexto, las brechas persisten incluso cuando el desempleo excede el nivel friccional. También se señala que, por diversas razones, los trabajadores pueden preferir ingresar al sector informal para evitar los costos de la protección social. Sin embargo, según este supuesto, la brecha de ingreso sería favorable a los trabajadores informales ${ }^{3}$. Otro argumento bastante común es que los menores ingresos de los trabajadores informales reflejan el valor de los beneficios no pecuniarios de estos puestos ${ }^{4}$, que los trabajadores independientes prefieren el trabajo autónomo, que los trabajadores secundarios —en su mayoría mujeres y jóvenes- eligen trabajos informales debido a la flexibilidad horaria y que los de mayor edad

\footnotetext{
${ }^{1}$ Además de Lewis (1954), otros estudios relevantes sobre la naturaleza de los mercados laborales duales son los de Harris y Todaro (1970); Fields (1975 y 1990), y Pradhan y Van Soest (1995).

2 Véanse Heckman y Sedlacek (1985); Dickens y Lang (1988); Evans y Leighton (1989); Pagés-Serra y Stampini (2007); Maloney (1999), y Pratap y Quintín (2006).

${ }^{3}$ La prima negativa formal indicaría que los ingresos de los puestos informales compensan la pérdida de protección social y la menor estabilidad.

4 Véase Killingsworth (1987); Magnac (1991); Maloney (1999), y Perry y otros (2007).
}

y los jóvenes poco calificados son compensados con la capacitación en el empleo.

También se cuestionan los procedimientos empíricos. Según la hipótesis de segmentación, las diferencias que se producen en el mercado para los trabajadores informales con respecto al resto de los trabajadores pueden dar lugar a que las ecuaciones de ingresos de ambos grupos tengan una forma funcional distinta. Hay datos que respaldan esta idea. Las funciones de ingreso que incorporan una variable para el sector formal o informal, así como las evaluaciones que calculan la diferencia entre las funciones de ingreso de diversos sectores, suelen dar como resultado modelos más parsimoniosos de la distribución de ingresos salariales que aquellas en las que solo se consideran variables de capital humano o de mercado laboral único. Sin embargo, se ha argumentado (véase, por ejemplo, Heckman y Hotz, 1986) que esto puede ser un simple reflejo del sesgo inducido por la composición selectiva de las muestras de trabajadores. De acuerdo con esta hipótesis, el mejor desempeño de los modelos de segmentación se debería sobre todo a la naturaleza paramétrica de las ecuaciones de ingresos utilizadas. En otras palabras, las estimaciones de mínimos cuadrados ordinarios (MCO) estarían sesgadas por la selectividad, dado que los individuos eligen el sector donde trabajar según los salarios que ofrece o las características productivas que tienden a variar de un sector a otro.

Para evitar las limitaciones de estas técnicas, existen otras metodologías que pueden resultar más adecuadas. En algunos enfoques alternativos se emplean métodos semiparamétricos o técnicas de corrección del sesgo de selección. Aun así, las primas estimadas con dichos procedimientos pueden surgir del efecto de variables no observadas (o no observables) - por ejemplo, las empresas formales pueden contratar a trabajadores más calificados o más inteligentes-, de modo que se requieren datos dinámicos a fin de tomarlas en cuenta. Específicamente, en este artículo se evalúa la existencia de una prima formal utilizando datos dinámicos y modelos semiparamétricos. Se comparan los cambios de los ingresos de aquellos trabajadores que pasan de la formalidad a la informalidad (y viceversa) con los de aquellos que permanecen en el mismo sector, formal o informal. Para los efectos de superar las restricciones emanadas de la composición selectiva de las muestras, las comparaciones se realizan entre trabajadores con 
probabilidades similares de transición sectorial basadas en las características observables.

Se emplean dos enfoques diferentes de la definición de informalidad: la definición tradicional utilizada por la Organización Internacional del Trabajo (OIT), basada en el número de trabajadores (el empleo en el sector informal), y la nueva definición que tiene en cuenta los aspectos legales y regulatorios (el empleo informal). Asimismo, a fin de profundizar en el análisis sobre las brechas de ingreso, en ocasiones se desagrega cada sector en algunos de sus componentes.

Los resultados obtenidos respaldan la hipótesis de segmentación del mercado laboral urbano de la Argentina: trabajadores que cuentan con probabilidades similares observables para transitar de un sector a otro obtienen diferentes ingresos.

Luego de la presente Introducción, el artículo presenta la siguiente estructura: en la sección II se procede a identificar la informalidad o sector informal de la economía, cuya extensión y reciente evolución se aborda en la sección III, para presentar en la sección IV las diferencias de ingresos entre los sectores formal e informal. En la sección $\mathrm{V}$ se lleva a cabo la estimación de la prima de formalidad mediante un modelo semiparamétrico con datos de panel. Finalmente, en la sexta sección se entregan las conclusiones a las que condujo el estudio.

\section{II}

\section{Identificación de la informalidad}

La informalidad — también llamada sector informal o economía informal - es una categoría utilizada para mejorar la descripción y el análisis del funcionamiento de la economía y, en particular, de los mercados laborales de los países en desarrollo. En la descripción original efectuada por la OIT $(1972)^{5}$ y sus modificaciones posteriores, principalmente en América Latina, se vincula la existencia de unidades informales de producción en los países de menor desarrollo con la incapacidad de sus economías en cuanto a crear suficientes puestos de trabajo para la fuerza laboral. Si bien en los países desarrollados esta situación conduciría al desempleo, en los países en desarrollo da lugar al surgimiento y la persistencia de una amplia concentración del empleo en unidades pequeñas de baja productividad. En ocasiones, ellas están compuestas simplemente por puestos de trabajo autónomos y, en otros casos, por asalariados que trabajan en pequeños establecimientos. Estas unidades de producción son capaces de subsistir explotando algunos "intersticios" del mercado o debido a sus bajas remuneraciones. Según esta visión tradicional de la oIT, las unidades informales suelen operar en actividades de fácil inserción y presentan una división poco clara entre el capital y la mano de obra.

Sin embargo, existen otras características vinculadas a la idea de la informalidad y otras explicaciones de su alcance, incluso en los países desarrollados. Por ejemplo,

\footnotetext{
5 Sin embargo, el término "informalidad" habría sido introducido por Hart (1973).
}

la informalidad se identifica con el no cumplimiento de las normas laborales y de otra índole (sobre todo con la evasión impositiva) por parte de ciertas empresas. Portes, Castells y Benton (1989) consideran que la relevancia cada vez mayor de la informalidad es el resultado de estrategias de reducción de costos, en un escenario altamente competitivo asociado con la nueva división internacional del trabajo que surgió a fines de la década de 1980. En este sentido, puede existir una informalidad oculta incluso en las grandes empresas si estas contratan trabajadores no registrados en el sistema de seguridad social. Otros autores —en particular De Soto (1987) opinan que el hecho de que en países menos desarrollados las unidades de menor tamaño tiendan a no registrarse se debe a que las regulaciones resultan complicadas y más adecuadas para empresas grandes. Claramente, según esta visión, la clasificación de las unidades se superpone en gran medida a la perspectiva tradicional de la OIT.

En cierto grado, en las recientes recomendaciones de la Conferencia Internacional de Estadísticos del Trabajo se toman en cuenta ambas perspectivas en lo que respecta a la definición de informalidad. En dichas recomendaciones se distingue el empleo en el sector informal (en términos generales, definido según la perspectiva tradicional de la OIT) del empleo informal, es decir, aquel que no está cubierto por la legislación laboral ${ }^{6}$. En este artículo, se adoptan ambas visiones con el fin de diferenciar la formalidad de la informalidad.

\footnotetext{
6 Véase Hussmanns (2005).
} 
Siguiendo una larga tradición en América Latina, se utiliza el tamaño de las empresas como criterio para distinguir entre establecimientos formales e informales. Se definen como trabajadores del sector formal aquellos que trabajan en firmas de seis empleados o más ${ }^{7}$. Se incluyen, además, las unidades del sector público —no solo la administración pública, sino todos los organismos nacionales, provinciales o municipales-. También se consideran parte del sector formal los trabajadores autónomos que han completado niveles superiores de educación, de manera que se establezca una distinción respecto de los trabajadores profesionales ${ }^{8}$.

\footnotetext{
${ }^{7}$ El tamaño de la empresa es la variable sustitutiva (proxy) habitual para la productividad cuando se utilizan datos de las encuestas de hogares. Sin embargo, puede haber pequeñas empresas con altos niveles de productividad (especialmente en el sector de la tecnología de la información), lo que se traduciría en una subestimación de la desigualdad salarial entre los sectores formal e informal. No obstante, la gran mayoría de las microempresas presentan baja productividad. ${ }^{8}$ Principalmente, abogados, médicos, contadores y otros profesionales autónomos similares.
}

En consecuencia, la clasificación de trabajadores informales abarca a los asalariados no registrados y a los trabajadores autónomos no profesionales. Un trabajador asalariado se considerará informal si no está registrado en el sistema de la seguridad social. Se incluye, además, a los empleados domésticos y a los beneficiarios de programas de empleo.

En el siguiente esquema se sintetizan las definiciones utilizadas:

\begin{tabular}{lcc}
\hline Sector de origen & $\begin{array}{c}\text { Empleo } \\
\text { informal }\end{array}$ & $\begin{array}{c}\text { Empleo en } \\
\text { el sector } \\
\text { informal }\end{array}$ \\
\hline $\begin{array}{l}\text { Trabajadores formales no asalariados } \\
\text { Trabajadores informales no asalariados }\end{array}$ & $\mathrm{x}$ & $\mathrm{x}$ \\
$\begin{array}{l}\text { Asalariados formales registrados } \\
\text { Asalariados formales no registrados }\end{array}$ & $\mathrm{x}$ & $\mathrm{x}$ \\
$\begin{array}{l}\text { Asalariados informales registrados } \\
\text { Asalariados informales no registrados }\end{array}$ & $\mathrm{x}$ & $\mathrm{x}$ \\
$\begin{array}{l}\text { Empleados domésticos } \\
\text { Trabajadores familiares }\end{array}$ & $\mathrm{x}$ & \\
Programas de empleo & $\mathrm{x}$ & \\
\hline
\end{tabular}

Fuente: Elaboración propia.

\section{III}

\section{Extensión y evolución reciente de la informalidad}

Las diferencias de ingresos entre los sectores formal e informal constituyen un tema relevante en la Argentina, dada la elevada informalidad existente en la estructura económica y laboral del país y su persistencia a lo largo del tiempo. En esta sección se examinan algunos datos que revelan el alcance de este sector.

El empleo informal — según la definición anteriorrepresentaba alrededor del 55\% del empleo urbano de la Argentina en 2005. Los asalariados no registrados constituían el $24 \%$ de esa cifra; los trabajadores autónomos informales, el $18 \%$, y los empleados domésticos, casi el 8\%; el resto de los trabajadores estaban inscritos en programas de empleo. Por otra parte, el empleo en el sector informal concentraba al $38 \%$ de los trabajadores, de los cuales un $20 \%$ trabajaba para pequeñas empresas (véase el cuadro 1).

A pesar de las dificultades que enfrentó el mercado laboral argentino durante las décadas de 1990 y 2000, cuando el desempleo creció del $9 \%$ en 1993 al 16\% en 2003, el tamaño relativo del empleo en el sector informal no cambió. Esto indica que las reformas estructurales que tuvieron lugar durante aquellos años afectaron también al sector informal, y que gran parte de este no consistía en actividades típicamente "informales" (Sánchez, Palmieri y Ferrero, 1976). Sin embargo, se registró un incremento significativo del número de asalariados no registrados, lo que explica la expansión - aunque modesta con relación a las dificultades laboralesdel empleo informal. Entre 1990 y 2010, la principal característica de la estructura del mercado laboral fue el aumento del número de asalariados no registrados (con la excepción de los empleados domésticos y los beneficiarios de programas de empleo). En 1993, estos trabajadores representaban el $29 \%$ del total de la fuerza laboral asalariada; en 2003, la cifra se había elevado al $41 \%$ (nuevamente, con la excepción de los empleados domésticos y los beneficiarios de programas de empleo).

Durante el período de crecimiento económico que comenzó a fines de 2002, el empleo en el sector informal y el empleo informal pasaron a representar un porcentaje 
menor de la tasa de empleo total, especialmente como resultado de la disminución del número de trabajadores autónomos. El número de asalariados no registrados se mantuvo estable (si bien se redujo su proporción con respecto al total de los asalariados debido al aumento de las ocupaciones registradas).

CUADRO 1

Argentina: estructura del mercado laboral, 1993-2005

(Todas las áreas urbanas encuestadas en porcentajes)

\begin{tabular}{|c|c|c|c|c|c|c|c|c|}
\hline & & $\begin{array}{c}\text { Oct } \\
1993\end{array}$ & $\begin{array}{c}\text { Oct } \\
1998\end{array}$ & $\begin{array}{c}\text { Oct } \\
2001\end{array}$ & $\begin{array}{l}\text { Mayo } \\
2003\end{array}$ & $\begin{array}{l}2^{\circ} \text { trimestre } \\
1993\end{array}$ & $\begin{array}{l}2^{\circ} \text { trimestre } \\
2003\end{array}$ & $\begin{array}{l}2^{\circ} \text { trimestre } \\
2005\end{array}$ \\
\hline 1 & Trabajadores no asalariados formales & 4,5 & 4,5 & 4,3 & 4,4 & 4,2 & 4,7 & 5,4 \\
\hline 2 & Trabajadores no asalariados informales & 23,4 & 19,4 & 21,2 & 20,7 & 20,8 & 19,6 & 18,4 \\
\hline 3 & Asalariados formales registrados & 38,7 & 39,1 & 37,9 & 34,2 & 30,1 & 30,9 & 34,1 \\
\hline 4 & Asalariados formales no registrados & 8,3 & 11,2 & 9,4 & 9,7 & 7,7 & 8,7 & 9,5 \\
\hline 5 & Asalariados informales registrados & 5,7 & 5,5 & 5,2 & 5,0 & 6,3 & 5,8 & 5,9 \\
\hline 6 & Asalariados informales no registrados & 10,3 & 11,8 & 12,1 & 11,2 & 14,5 & 14,4 & 14,0 \\
\hline 7 & Empleados domésticos & 7,9 & 7,3 & 7,6 & 6,8 & 7,8 & 7,5 & 7,7 \\
\hline 8 & Trabajadores familiares & 1,3 & 1,3 & 0,8 & 1,0 & 1,6 & 1,7 & 1,2 \\
\hline \multirow[t]{3}{*}{9} & Beneficiarios de programas de empleo & 0,0 & 0,0 & 1,4 & 7,0 & 7,0 & 6,7 & 3,7 \\
\hline & Total & 100 & 100 & 100 & 100 & 100 & 100 & 100 \\
\hline & Con programas de empleo & & & & & & & \\
\hline$(2+4+6+7+8+9)$ & Empleo informal & 51,1 & 50,9 & 52,5 & 56,4 & 59,4 & 58,6 & 54,6 \\
\hline \multirow[t]{5}{*}{$(2+5+6)$} & Empleo en el sector informal & 39,4 & 36,7 & 38,6 & 36,9 & 41,6 & 39,7 & 38,4 \\
\hline & $\begin{array}{l}\text { Asalariados no registrados + empleados } \\
\text { domésticos }+ \text { beneficiarios programas de } \\
\text { empleo }\end{array}$ & 26,5 & 30,2 & 30,5 & 34,7 & 37,0 & 37,4 & 34,9 \\
\hline & $\begin{array}{l}\text { Asalariados no registrados }+ \text { beneficiarios } \\
\text { programas de empleo }\end{array}$ & 18,6 & 23,0 & 22,9 & 27,9 & 29,2 & 29,9 & 27,2 \\
\hline & Asalariados no registrados & 18,6 & 23,0 & 21,5 & 20,9 & 22,1 & 23,1 & 23,6 \\
\hline & Sin programas de empleo & & & & & & & \\
\hline$(2+4+6+7+8)$ & Empleo informal & 51,1 & 50,9 & 51,8 & 53,1 & 56,3 & 55,6 & 52,8 \\
\hline \multirow[t]{3}{*}{$(2+5+6)$} & Empleo en el sector informal & 39,4 & 36,7 & 39,1 & 39,7 & 44,7 & 42,6 & 39,8 \\
\hline & $\begin{array}{l}\text { Asalariados no registrados + empleados } \\
\text { domésticos }\end{array}$ & 26,5 & 30,2 & 29,5 & 29,8 & 32,2 & 32,8 & 32,4 \\
\hline & Asalariados no registrados & 18,6 & 23,0 & 21,8 & 22,5 & 23,8 & 24,8 & 24,5 \\
\hline
\end{tabular}

Fuente: Elaboración propia sobre la base de datos de las encuestas permanentes de hogares. 


\section{IV \\ Diferencias de ingresos entre los sectores formal e informal: datos y anteriores resultados}

La diferencia entre los ingresos medios de los diferentes grupos de trabajadores y otras categorías desagregadas puede observarse en el cuadro 2. En promedio, los ingresos por hora de los trabajadores del sector informal son un $47 \%$ inferiores a los de aquellos que trabajan en el sector formal. Las diferencias entre el empleo informal y el formal y entre los asalariados no registrados y los registrados son del $48 \%$ y el $42 \%$, respectivamente. Las brechas se amplían al comparar los ingresos mensuales, dado que la informalidad se vincula al subempleo (véase el cuadro 2).

En algunos estudios, los efectos de la informalidad se distinguen de aquellos causados por otras variables determinantes del ingreso mediante funciones de ingreso estimadas a partir de datos transversales. Por ejemplo, Beccaria y Groisman (2008), sobre la base de datos de 2005 , calculan que existe una brecha de alrededor del $40 \%$ entre los ingresos por hora de los trabajadores asalariados, mientras que entre el empleo en el sector formal y el del sector informal la brecha es menor. Según Perry y otros (2007), la brecha entre los trabajadores registrados y los no registrados es de un 35\%, en tanto que Pratap y Quintín (2006), sobre la base de datos específicos del Gran Buenos Aires entre 1993 y 1995, calculan que (dicha brecha) se ubica entre un $22 \%$ y un $28 \%$ entre los asalariados registrados y los no registrados (dependiendo del modelo). Estas cifras son similares a las estimadas por Beccaria y Groisman (2008) respecto de 1993.

Este enfoque, basado en funciones de ingreso, se asienta sobre suposiciones paramétricas. El problema metodológico básico, que no se limita a esta cuestión, es que los modelos segmentados o duales pueden encajar mejor por el mero hecho de que las funciones de ingreso son no lineales. Si la función de ingreso subyacente es lo bastante no lineal, los análisis de segmentación resultarán más parsimoniosos. Además, se ha sugerido que la corrección de Heckman no sirve para limitar el sesgo de selección.

Argentina: ingresos y horas de trabajo, segundo trimestre de 2005

(Todas las áreas urbanas encuestadas)

\begin{tabular}{|c|c|c|c|c|c|c|}
\hline & $\begin{array}{l}\text { Horas de trabajo } \\
\text { semanales }\end{array}$ & $\begin{array}{l}\text { Ingresos } \\
\text { mensuales } \\
\text { (en pesos } \\
\text { argentinos) }\end{array}$ & $\begin{array}{l}\text { Ingresos por } \\
\text { hora (en pesos } \\
\text { argentinos) }\end{array}$ & $\begin{array}{l}\text { Horas de trabajo } \\
\text { semanales }\end{array}$ & $\begin{array}{l}\text { Ingresos } \\
\text { mensuales } \\
\text { (en pesos } \\
\text { argentinos) }\end{array}$ & $\begin{array}{l}\text { Ingresos por } \\
\text { hora (en pesos } \\
\text { argentinos) }\end{array}$ \\
\hline & \multicolumn{3}{|c|}{ Empleo sector informal } & \multicolumn{3}{|c|}{ Empleo sector formal } \\
\hline Nivel bajo de educación & 42,0 & 486,8 & 3,3 & 47,7 & 776,2 & 4,1 \\
\hline Nivel medio de educación & 42,5 & 711,8 & 4,5 & 43,1 & 1047,8 & 6,2 \\
\hline Nivel alto de educación & 38,8 & 936,1 & 6,1 & 37,8 & 1566,9 & 11,3 \\
\hline \multirow[t]{2}{*}{ Promedio } & 42,1 & 582,1 & 3,8 & 42,9 & 1128,2 & 7,2 \\
\hline & \multicolumn{3}{|c|}{ Empleo informal } & \multicolumn{3}{|c|}{ Empleo formal } \\
\hline Nivel bajo de educación & 38,1 & 417,8 & 3,2 & 48,4 & 897,1 & 4,7 \\
\hline Nivel medio de educación & 39,3 & 626,6 & 4,4 & 44,7 & 1112,4 & 6,3 \\
\hline Nivel alto de educación & 36,1 & 1092,2 & 10,3 & 38,1 & 1567,4 & 10,9 \\
\hline \multirow[t]{2}{*}{ Promedio } & 38,4 & 510,6 & 3,8 & 43,5 & 1205,6 & 7,4 \\
\hline & \multicolumn{3}{|c|}{ Asalariados no registrados } & \multicolumn{3}{|c|}{ Asalariados registrados } \\
\hline Nivel bajo de educación & 43,1 & 432,1 & 2,6 & 48,3 & 881,9 & 4,6 \\
\hline Nivel medio de educación & 38,0 & 531,3 & 3,8 & 44,5 & 1070,7 & 6,1 \\
\hline Nivel alto de educación & 36,7 & 1155,1 & 10,9 & 36,8 & 1435,8 & 9,6 \\
\hline Promedio & 40,8 & 530,8 & 3,8 & 43,7 & 1104,8 & 6,5 \\
\hline
\end{tabular}

Fuente: Elaboración propia sobre la base de datos de las encuestas permanentes de hogares. 
Por este motivo, en varios estudios de diferentes países se utilizan métodos semiparamétricos ${ }^{9}$. Beccaria y Groisman (2008) aplican al caso de la Argentina este enfoque basado en métodos de regresiones por cuantiles y de puntaje de propensión. Los resultados de la prima de formalidad son similares a los que se incluyeron en el artículo de 2008 antes mencionado, en el que se emplearon funciones de ingreso. Pratap y Quintín (2006) también utilizan el cotejo por puntaje de propensión, un enfoque alternativo que modera las suposiciones paramétricas de las funciones de ingreso. En este caso, los resultados muestran que la prima de formalidad desaparece, contradiciendo lo expresado en aquellos estudios en que se habían utilizado funciones de ingreso. La brecha de ingresos entre los empleados registrados y los no registrados solo resurge una vez que se comparan los salarios de los empleados de empresas de distintos tamaños (es decir, cuando se excluye el tamaño de entre las variables empleadas para estimar los puntajes de propensión).

Al utilizar datos transversales no se puede controlar adecuadamente el efecto de las variables no observadas. Una forma común de abordar este problema es mediante la utilización de modelos de efecto fijo. Pratap y Quintín (2006) observan una regresión estándar de efecto fijo sobre el logaritmo de los salarios reales por hora, que

\footnotetext{
${ }^{9}$ Por ejemplo, Maloney (1999) en el caso de México; Packard (2007) en el caso de Chile, y Perry y otros (2007), que examinan varios países latinoamericanos.
}

da como resultado una importante prima positiva del sector formal.

Otra alternativa es emplear modelos semiparamétricos con datos de panel. Al aplicar el cotejo por puntaje de propensión a los datos de panel, es posible calcular si la variación de los ingresos de los trabajadores formales en un sector determinado es similar a la de los trabajadores formales que han pasado a desempeñar una ocupación informal. La ausencia de diferencias indicaría que el mercado laboral no está segmentado entre el sector formal y el informal. Pratap y Quintín (2006) aplican este enfoque, pero determinan que sus resultados no son concluyentes, debido - entre otras causas - al reducido tamaño de la muestra. En efecto, los datos que utilizaron se limitaban al Gran Buenos Aires en el período 1993-1995.

Perry y otros (2007) estudian las estimaciones de la brecha de ingresos entre los sectores formal e informal que efectúan Arias y Khamis, con un enfoque de puntaje de propensión aplicado a datos de panel. Estas estimaciones revelan grandes diferencias positivas entre los ingresos de los asalariados registrados y los de los asalariados no registrados, mientras que — si se comparan los ingresos de los trabajadores asalariados con los de los no asalariados- estas diferencias se vuelven poco significativas o incluso negativas.

En la siguiente sección se pretende ampliar el trabajo de Pratap y Quintín (2006) por medio de un conjunto más amplio de datos de panel (que abarca todas las zonas urbanas encuestadas y períodos más largos: específicamente, de 1996 a 2003 y de 2004 a 2006) y con la incorporación de las dos definiciones de informalidad mencionadas en la sección II.

\section{V}

\section{Estimación de la prima de formalidad mediante un modelo semiparamétrico con datos de panel}

\section{Método}

En este estudio se adopta un enfoque semiparamétrico para determinar si hay diferencias de ingresos entre el sector formal y el informal. Se aplica el método de puntaje de propensión para comparar los ingresos de los trabajadores formales e informales con similares características, a fin de determinar si existe alguna diferencia. Para identificar a los trabajadores "más similares" se atribuye un puntaje a cada observación. El puntaje se basa en la probabilidad de que una persona trabaje en el sector formal según los modelos de probabilidades (función probit), teniendo en cuenta las variables relevantes disponibles en el conjunto de datos. De este modo, se calcula el promedio de las diferencias entre personas con puntajes similares $-\alpha-$ utilizando la siguiente fórmula: 


$$
\alpha^{M}=\frac{1}{N_{F}} \sum_{i \in I}\left(w_{i}^{F} \sum_{j \in I} \eta_{i j} w_{j}^{I}\right)
$$

donde $F$ representa el sector formal, $I$ el sector informal, $N_{F}$ es el número de trabajadores formales y $\eta_{i j}$ su ponderación comparativa.

La ponderación de cada observación puede determinarse mediante diferentes algoritmos de cotejo: es frecuente utilizar el método del vecino más próximo, donde $\eta_{i j}$ es igual a 1 para $\min \left|p_{i}-p_{j}\right|$ e igual a 0 para otros, $\mathrm{y}$ $p_{k}$ representa el puntaje de propensión del trabajador $k$. Un algoritmo alternativo es el emparejamiento por radio, que establece un radio máximo de puntaje de propensión y considera el promedio de los ingresos de todos los trabajadores incluidos en ese radio. El estimador de Kernel es otra variante por la que los ingresos de los trabajadores formales $(i)$ se comparan con el promedio ponderado de los ingresos de los trabajadores informales $(j)$, y en la que la ponderación está inversamente relacionada con la distancia existente entre los puntajes de $i$ y $j$, es decir, $\left|p_{i}-p_{j}\right|$.

El método semiparamétrico no requiere suposiciones en forma de funciones de ingresos y limita las comparaciones a los trabajadores cuya similitud es observable.

Si bien este método soluciona el problema del sesgo de la selección, como se considera habitualmente, no resuelve otra dificultad común de los métodos paramétricos: el efecto de las variables no observadas. Algo que se suele criticar de los estudios de segmentación es que no toman en cuenta el efecto de variables que generalmente no se observan y que afectan a los ingresos. Por este motivo, al igual que en otros estudios recientes, se ha adoptado el enfoque del puntaje de propensión, no para comparar los ingresos de los trabajadores formales e informales en un determinado momento, sino con el propósito de evaluar las diferencias de ingresos a lo largo del tiempo. Por medio de datos de panel, se contrasta la diferencia de los ingresos de los trabajadores que habían pasado de la formalidad a la informalidad entre el período $0 \mathrm{y}$ el período $t$ con aquellos que permanecieron en el sector formal en ambos períodos. De igual manera, se compara la diferencia de ingresos de los trabajadores informales que accedieron a un puesto formal con la de aquellos trabajadores que permanecieron en el sector informal. La diferencia media se calcula de la siguiente manera:

$$
\alpha^{M}=\frac{1}{N_{F}} \sum_{i \in F}\left[\left({ }_{t} w_{i}^{F}-{ }_{0} w_{i}^{F}\right)-\sum_{j \in I} \eta_{i j}\left({ }_{t} w_{i}^{F}-{ }_{0} w_{i}^{I}\right)\right]
$$

donde 0 y $t$ denotan los dos períodos considerados.
En este caso, en lugar de referirse a la probabilidad de trabajar en un puesto informal, el puntaje indica la probabilidad de que un trabajador con un puesto formal en el primer período cambie a un puesto informal en el segundo, y viceversa. Las probabilidades se calculan mediante una función probit en que se consideran las siguientes variables independientes: edad, edad al cuadrado, género, educación y posición en el hogar.

La estructura de panel de los datos modera parcialmente el posible impacto de las características no observables en los ingresos, pero no modera el efecto que pueden tener en las transiciones entre sectores.

El método del vecino más próximo se emplea para ponderar las observaciones, es decir, el cambio del nivel de ingresos de cada trabajador que permanece en la formalidad comparado con las diferencias de ingresos de los trabajadores que transitan de la formalidad a la informalidad y tienen el mismo puntaje de propensión o el más cercano.

Se comparan los resultados con los obtenidos mediante funciones de ingresos estándares (MCO y el modelo de efecto fijo) aplicadas a los mismos datos de los dos períodos considerados. Estos modelos incluyen una variable ficticia para la informalidad. El modelo de efectos fijos modera parcialmente el impacto de los determinantes fijos y no observados de los ingresos.

\section{Datos}

Si bien en la Argentina no se realizan encuestas longitudinales, la encuesta permanente de hogares que lleva a cabo regularmente el Instituto Nacional de Estadística y Censos (INDEC) ${ }^{10}$ proporciona datos de ese tipo utilizando una muestra de panel rotativa: los hogares son entrevistados en cuatro ocasiones sucesivas, de modo que, al comparar la situación de un individuo en estas cuatro etapas, es posible inferir los cambios experimentados en un número de variables, incluido el ingreso.

La muestra de la Encuesta Permanente de Hogares se compone de cuatro grupos de rotación. Hasta 2003, las encuestas se realizaban dos veces por año, en mayo y octubre, de manera que un grupo comenzaba y otro concluía en cada una de las dos sesiones del año. En consecuencia, en cada ocasión se renovaba el $25 \%$ de la muestra, por lo que podía compararse el $75 \%$

\footnotetext{
10 Véase una descripción de la metodología aplicada en las encuestas permanentes de hogares [en línea] www.indec.gov.ar. El esquema de las encuestas cambió sustancialmente en 2003.
} 
de los casos entre dos etapas sucesivas o el $50 \%$ en un período de 12 meses. Desde 2003, cuando se modificó el esquema de rotación y las encuestas comenzaron a efectuarse trimestralmente, los hogares son encuestados en dos trimestres consecutivos. Luego se retiran durante dos trimestres consecutivos antes de reincorporarse en dos trimestres más. Así, es posible comparar el 50\% de la muestra entre los mismos trimestres de dos años consecutivos. Si bien las encuestas se vienen realizando desde 1974, solo están disponibles los microdatos de las 28 ciudades de las encuestas que se realizaron a partir de 1996.

En el presente estudio se analizan dos subperíodos distintos: el comprendido entre 1996 y 2003, cuando se elevó el número de trabajadores asalariados no formales, y el lapso de 2004 a 2006, en el que el tamaño del sector informal se mantuvo sin cambios. Estos dos subperíodos también corresponden al mencionado cambio en el método de recolección de datos de la Encuesta Permanente de Hogares.

Para el subperíodo de 1996 a 2003 se utilizaron datos de las encuestas realizadas en el mes de mayo de cada año, de tal forma que se compara la situación del mismo hogar o individuo en dos años sucesivos. Entre 2004 y 2006 se manejan datos que registran los cambios ocurridos en los primeros seis meses (primer y segundo trimestres) de dos años consecutivos. Así, se miden los cambios comparando el $50 \%$ de la muestra en ambos subperíodos. Sin embargo, cabe señalar que la proporción de hogares e individuos que vuelven a ser entrevistados es en realidad menor; esto se debe a una disminución de la muestra atribuible a varias causas, como por ejemplo, que los hogares abandonen el panel o cambien de dirección, o dificultades surgidas en el trabajo de campo.

Dado que es más beneficioso trabajar con un gran número de casos, se agregaron los grupos de rotación que fueron ingresados a la muestra en cada momento. De esta manera, las bases de datos incluyen cambios ocurridos en distintos períodos.

\section{Resultados principales}

En el cuadro 3 se presentan los parámetros de las funciones de ingreso calculados por medio del modelo de efecto fijo para medir el impacto de las variables no observadas, como también los parámetros basados en MCO. Puede observarse que las brechas de ingresos entre la formalidad y la informalidad derivadas de ambos modelos son distintas a 0 . Sin embargo, la prima formal resulta menor en el modelo de efecto fijo, lo que sugiere que este efecto es el responsable de parte de las diferencias que suelen registrarse.

No obstante, como se ha indicado anteriormente, es posible que exista un sesgo en las brechas calculadas a partir de las funciones de ingreso, incluso las que tienen en cuenta el efecto fijo, debido a la naturaleza paramétrica de la función de ingresos estimada mediante MCO. Por este motivo se ha utilizado un método semiparamétrico, basado en puntajes de propensión aplicados a datos de panel, que contempla las variables no observadas.

Los puntajes de propensión estimados para los tres enfoques considerados -empleo informal, empleo en el sector informal y asalariados no registrados-dejan ver que en todos los casos existe una correlación negativa entre la transición de un puesto de trabajo formal a uno informal y la edad. De manera similar, según la mayoría de los modelos probit, los trabajadores con un alto nivel de educación y las mujeres en general tienen menos probabilidades de ingresar al sector informal (véase el cuadro 4). Si bien lo contrario no siempre se da, los trabajadores que tienen mayores posibilidades de transitar de la informalidad a la formalidad son aquellos cuyo nivel de educación es más alto. Para los asalariados se calcularon modelos probit en los que se excluyó o incluyó el tamaño de la empresa como variable independiente. El modelo en que se incluyó el tamaño de la empresa denota que los asalariados de las grandes firmas tienen una menor probabilidad de no estar registrados, o una mayor probabilidad de estar registrados. Por lo tanto, los resultados obtenidos son los esperados: los hombres jóvenes y los trabajadores con menor nivel de educación tienen más probabilidades de transitar de la formalidad a la informalidad, mientras que los asalariados de pequeñas empresas tienen una mayor probabilidad de soportar condiciones de trabajo precarias.

En cuanto a la prima del sector formal, los resultados obtenidos mediante el método de puntajes de propensión aplicado a datos de panel parecen indicar que existe una segmentación de los salarios en ambos contextos estudiados (véase el cuadro 5, panel 1), esto es, que los ingresos de los trabajadores que pasan de una ocupación informal a una formal se incrementan más (o se reducen menos) que los ingresos de aquellos que permanecen en el sector informal. Si se comparan los salarios de los trabajadores que han pasado de una ocupación formal a una informal con los salarios de aquellos que han permanecido en el sector formal, se obtienen resultados simétricos. Esto se registra tanto en el período comprendido entre 1996 y 2003 como entre 2004 y 2006, si bien en este último lapso aumenta la brecha de ingresos, especialmente en el caso del empleo informal. 
CUADRO 3

Argentina: funciones de ingreso, 1996-2003 y 2004-2006

(Todas las áreas urbanas encuestadas)

\begin{tabular}{|c|c|c|c|c|c|c|c|c|}
\hline & \multicolumn{4}{|c|}{$1996-2003$} & \multicolumn{4}{|c|}{ 2004-2006 } \\
\hline & \multicolumn{2}{|c|}{$\mathrm{MCO}$} & \multicolumn{2}{|c|}{ MCO-efecto fijo } & \multicolumn{2}{|c|}{$\mathrm{MCO}$} & \multicolumn{2}{|c|}{ MCO-efecto fijo } \\
\hline & $\mathrm{b}$ & Sig & $\mathrm{b}$ & Sig & $\mathrm{b}$ & Sig & $\mathrm{b}$ & Sig \\
\hline \multicolumn{9}{|l|}{ A. Empleo informal } \\
\hline Jefe de familia & 0,104 & 0,000 & & & 0,109 & 0,000 & & \\
\hline Sexo & 0,182 & 0,000 & & & 0,213 & 0,000 & & \\
\hline Edad & 0,051 & 0,000 & 0,000 & 0,972 & 0,043 & 0,000 & 0,026 & 0,000 \\
\hline Edad al cuadrado & $-0,001$ & 0,000 & 0,000 & 0,115 & 0,000 & 0,000 & 0,000 & 0,000 \\
\hline Nivel bajo de educación & $-0,478$ & 0,000 & & & $-0,378$ & 0,000 & & \\
\hline Horas & $-0,013$ & 0,000 & $-0,017$ & 0,000 & $-0,014$ & 0,000 & $-0,017$ & 0,000 \\
\hline Sector público & 0,053 & 0,000 & 0,033 & 0,014 & 0,010 & 0,035 & 0,011 & 0,470 \\
\hline Servicios sociales & 0,021 & 0,000 & 0,038 & 0,001 & 0,027 & 0,000 & 0,033 & 0,047 \\
\hline Transporte & 0,063 & 0,000 & 0,056 & 0,001 & 0,055 & 0,000 & 0,072 & 0,001 \\
\hline Comercio & $-0,084$ & 0,000 & $-0,071$ & 0,000 & $-0,107$ & 0,000 & $-0,020$ & 0,155 \\
\hline Construcción & $-0,205$ & 0,000 & 0,001 & 0,953 & $-0,054$ & 0,000 & 0,071 & 0,000 \\
\hline Industria & $-0,063$ & 0,000 & $-0,003$ & 0,805 & $-0,053$ & 0,000 & 0,014 & 0,369 \\
\hline Gran Buenos Aires & $-0,059$ & 0,000 & & & $-0,210$ & 0,000 & & \\
\hline Noroeste argentino & $-0,471$ & 0,000 & & & $-0,614$ & 0,000 & & \\
\hline Nordeste argentino & $-0,565$ & 0,000 & & & $-0,672$ & 0,000 & & \\
\hline Pampas & $-0,309$ & 0,000 & & & $-0,345$ & 0,000 & & \\
\hline Cuyo & $-0,427$ & 0,000 & & & $-0,472$ & 0,000 & & \\
\hline Empleo informal & $-0,414$ & 0,000 & $-0,169$ & 0,000 & $-0,629$ & 0,000 & $-0,319$ & 0,000 \\
\hline Año ficticio 1 & $-0,016$ & 0,001 & & & 0,050 & 0,000 & & \\
\hline Año ficticio 2 & $-0,010$ & 0,049 & & & 0,100 & 0,000 & & \\
\hline Año ficticio 3 & $-0,046$ & 0,000 & & & 0,150 & 0,000 & & \\
\hline Año ficticio 4 & $-0,074$ & 0,000 & & & 0,202 & 0,000 & 1,405 & 0,000 \\
\hline Año ficticio 5 & $-0,105$ & 0,000 & & & & & & \\
\hline Año ficticio 6 & $-0,340$ & 0,000 & & & & & & \\
\hline Año ficticio 7 & $-0,476$ & 0,000 & & & & & & \\
\hline Constante & 1,608 & 0,000 & 2,204 & 0,000 & 1,338 & 0,000 & & \\
\hline$\underline{\mathrm{R} \text { al cuadrado }}$ & 0,440 & & 0,156 & & 0,488 & & 0,233 & \\
\hline \multicolumn{9}{|l|}{ B. Empleo sector informal } \\
\hline Jefe de familia & 0,115 & 0,000 & & & 0,142 & 0,000 & & \\
\hline Sexo & 0,088 & 0,000 & & & 0,088 & 0,000 & & \\
\hline Edad & 0,056 & 0,000 & 0,000 & 0,893 & 0,058 & 0,000 & 0,029 & 0,000 \\
\hline Edad al cuadrado & $-0,001$ & 0,000 & 0,000 & 0,091 & $-0,001$ & 0,000 & 0,000 & 0,000 \\
\hline Nivel bajo de educación & $-0,474$ & 0,000 & & & $-0,393$ & 0,000 & & \\
\hline Horas & $-0,013$ & 0,000 & $-0,017$ & 0,000 & $-0,012$ & 0,000 & $-0,017$ & 0,000 \\
\hline Sector público & 0,029 & 0,000 & 0,069 & 0,000 & 0,048 & 0,000 & 0,046 & 0,002 \\
\hline Servicios sociales & $-0,074$ & 0,000 & 0,062 & 0,000 & $-0,067$ & 0,000 & 0,054 & 0,001 \\
\hline Transporte & $-0,099$ & 0,000 & 0,063 & 0,000 & $-0,098$ & 0,000 & 0,068 & 0,002 \\
\hline Comercio & $-0,166$ & 0,000 & $-0,056$ & 0,000 & $-0,195$ & 0,000 & $-0,010$ & 0,458 \\
\hline Construcción & $-0,353$ & 0,000 & 0,005 & 0,710 & $-0,200$ & 0,000 & 0,064 & 0,000 \\
\hline Industria & $-0,131$ & 0,000 & 0,019 & 0,144 & $-0,094$ & 0,000 & 0,030 & 0,067 \\
\hline Gran Buenos Aires & $-0,138$ & 0,000 & & & $-0,275$ & 0,000 & & \\
\hline Noroeste argentino & $-0,488$ & 0,000 & & & $-0,648$ & 0,000 & & \\
\hline Nordeste argentino & $-0,558$ & 0,000 & & & $-0,683$ & 0,000 & & \\
\hline Pampas & $-0,360$ & 0,000 & & & $-0,386$ & 0,000 & & \\
\hline Cuyo & $-0,459$ & 0,000 & & & $-0,527$ & 0,000 & & \\
\hline Empleo sector informal & $-0,262$ & 0,000 & $-0,044$ & 0,000 & $-0,370$ & 0,000 & $-0,081$ & 0,000 \\
\hline Año ficticio 1 & $-0,014$ & 0,013 & & & 0,062 & 0,000 & & \\
\hline Año ficticio 2 & $-0,015$ & 0,007 & & & 0,122 & 0,000 & & \\
\hline Año ficticio 3 & $-0,041$ & 0,000 & & & 0,187 & 0,000 & & \\
\hline Año ficticio 4 & $-0,064$ & 0,000 & & & 0,245 & 0,000 & & \\
\hline Año ficticio 5 & $-0,089$ & 0,000 & & & & & & \\
\hline Año ficticio 6 & $-0,281$ & 0,000 & & & & & & \\
\hline Año ficticio 7 & $-0,426$ & 0,000 & & & & & & \\
\hline Constante & 1,543 & 0,000 & 2,104 & 0,000 & 1,020 & 0,000 & 1,172 & 0,000 \\
\hline $\mathrm{R}$ al cuadrado & 0,455 & & 0,107 & & 0,486 & & 0,089 & \\
\hline
\end{tabular}


Cuadro 3 (conclusión)

\begin{tabular}{|c|c|c|c|c|c|c|c|c|}
\hline & \multicolumn{4}{|c|}{ 1996-2003 } & \multicolumn{4}{|c|}{ 2004-2006 } \\
\hline & \multicolumn{2}{|c|}{$\mathrm{MCO}$} & \multicolumn{2}{|c|}{ MCO-efecto fijo } & \multicolumn{2}{|c|}{$\mathrm{MCO}$} & \multicolumn{2}{|c|}{ MCO-efecto fijo } \\
\hline & $\mathrm{b}$ & Sig & $\mathrm{b}$ & Sig & $\mathrm{b}$ & Sig & $\mathrm{b}$ & Sig \\
\hline \multicolumn{9}{|l|}{ C. Asalariados } \\
\hline Jefe de familia & 0,097 & 0,000 & & & 0,101 & 0,000 & & \\
\hline Edad & 0,043 & 0,000 & 0,000 & 0,937 & 0,048 & 0,000 & 0,027 & 0,000 \\
\hline Edad al cuadrado & 0,000 & 0,000 & 0,000 & 0,136 & 0,000 & 0,000 & 0,000 & 0,000 \\
\hline Nivel bajo de educación & $-0,451$ & 0,000 & & & $-0,494$ & 0,000 & & \\
\hline Horas & $-0,014$ & 0,000 & $-0,017$ & 0,000 & $-0,012$ & 0,000 & $-0,017$ & 0,000 \\
\hline Sector público & $-0,041$ & 0,000 & 0,030 & 0,027 & 0,025 & 0,000 & 0,008 & 0,612 \\
\hline Servicios sociales & $-0,077$ & 0,000 & 0,048 & 0,000 & 0,109 & 0,000 & 0,049 & 0,004 \\
\hline Transporte & $-0,048$ & 0,000 & 0,062 & 0,000 & 0,065 & 0,000 & 0,078 & 0,000 \\
\hline Comercio & $-0,156$ & 0,000 & $-0,056$ & 0,000 & $-0,081$ & 0,000 & 0,000 & 0,975 \\
\hline Industria & $-0,143$ & 0,000 & 0,008 & 0,553 & $-0,028$ & 0,000 & 0,030 & 0,063 \\
\hline Gran Buenos Aires & $-0,119$ & 0,000 & & & $-0,241$ & 0,000 & & \\
\hline Noroeste argentino & $-0,448$ & 0,000 & & & $-0,688$ & 0,000 & & \\
\hline Nordeste argentino & $-0,512$ & 0,000 & & & $-0,727$ & 0,000 & & \\
\hline Pampas & $-0,337$ & 0,000 & & & $-0,372$ & 0,000 & & \\
\hline Cuyo & $-0,428$ & 0,000 & & & $-0,559$ & 0,000 & & \\
\hline Asalariados no registrados & $-0,363$ & 0,000 & $-0,083$ & 0,000 & $-0,232$ & 0,000 & $-0,113$ & 0,000 \\
\hline Año ficticio 1 & $-0,006$ & 0,258 & & & 0,061 & 0,000 & & \\
\hline Año ficticio 2 & $-0,002$ & 0,702 & & & 0,111 & 0,000 & & \\
\hline Año ficticio 3 & $-0,025$ & 0,000 & & & 0,168 & 0,000 & & \\
\hline Año ficticio 4 & $-0,046$ & 0,000 & & & 0,225 & 0,000 & & \\
\hline Año ficticio 5 & $-0,064$ & 0,000 & & & & & & \\
\hline Año ficticio 6 & $-0,266$ & 0,000 & & & & & & \\
\hline Año ficticio 7 & $-0,399$ & 0,000 & & & & & & \\
\hline $\mathrm{T} 1$ & 0,112 & 0,000 & 0,085 & 0,000 & 0,254 & 0,000 & 0,087 & 0,000 \\
\hline T 2 & 0,154 & 0,000 & 0,099 & 0,000 & 0,384 & 0,000 & 0,145 & 0,000 \\
\hline Constante & 1,726 & 0,000 & 2,090 & 0,000 & 0,746 & 0,000 & 1,170 & 0,000 \\
\hline Regresión al cuadrado & 0,496 & & 0,132 & & 0,433 & & 0,129 & \\
\hline
\end{tabular}

Fuente: Elaboración propia sobre la base de datos de las encuestas permanentes de hogares.

Nota: MCo: Mínimos cuadrados ordinarios; Sig: Significación; b: Coeficiente de regresión; T 1, 2, 3 y 4: Rangos de tamaño.

CUADRO 4

Argentina: resultados de la estimación probit de los puntajes de propensión, 1996-2003 y 2004-2006

(Todas las áreas urbanas encuestadas)

\begin{tabular}{|c|c|c|c|c|}
\hline & \multicolumn{2}{|c|}{$1996-2003$} & \multicolumn{2}{|c|}{ 2004-2006 } \\
\hline & Coeficiente & Sig & Coeficiente & Sig \\
\hline Edad & $-0,061$ & 0,000 & $-0,049$ & 0,000 \\
\hline Edad al cuadrado & 0,001 & 0,000 & 0,000 & 0,000 \\
\hline Sexo masculino & 0,246 & 0,000 & 0,183 & 0,000 \\
\hline Nivel bajo de educación & 0,328 & 0,000 & 0,307 & 0,000 \\
\hline Jefe de familia & $-0,058$ & 0,092 & $-0,052$ & 0,251 \\
\hline
\end{tabular}


Cuadro 4 (conclusión)

\begin{tabular}{|c|c|c|c|c|}
\hline & 19 & & 200 & \\
\hline & Coeficiente & Sig & Coeficiente & Sig \\
\hline Del empleo informal al emp & & & & \\
\hline $\begin{array}{l}\text { Edad } \\
\text { Edad al cuadrado } \\
\text { Sexo masculino } \\
\text { Nivel bajo de educación } \\
\text { Jefe de familia } \\
\end{array}$ & $\begin{array}{r}0,002 \\
0,000 \\
0,028 \\
-0,436 \\
0,036 \\
\end{array}$ & $\begin{array}{l}0,691 \\
0,028 \\
0,341 \\
0,000 \\
0,248 \\
\end{array}$ & $\begin{array}{r}0,005 \\
0,000 \\
0,056 \\
-0,491 \\
-0,018 \\
\end{array}$ & $\begin{array}{l}0,474 \\
0,030 \\
0,102 \\
0,000 \\
0,618 \\
\end{array}$ \\
\hline Del empleo en el sector for & & & & \\
\hline $\begin{array}{l}\text { Edad } \\
\text { Edad al cuadrado } \\
\text { Sexo masculino } \\
\text { Nivel bajo de educación } \\
\text { Jefe de familia }\end{array}$ & $\begin{array}{r}-0,047 \\
0,001 \\
0,257 \\
0,304 \\
-0,034 \\
\end{array}$ & $\begin{array}{l}0,000 \\
0,000 \\
0,000 \\
0,000 \\
0,252 \\
\end{array}$ & $\begin{array}{r}-0,057 \\
0,001 \\
0,203 \\
-0,400 \\
-0,053 \\
\end{array}$ & $\begin{array}{l}0,000 \\
0,000 \\
0,000 \\
0,000 \\
0,157\end{array}$ \\
\hline Del empleo en el sector info & & & & \\
\hline $\begin{array}{l}\text { Edad } \\
\text { Edad al cuadrado } \\
\text { Sexo masculino } \\
\text { Nivel bajo de educación } \\
\text { Jefe de familia } \\
\end{array}$ & $\begin{array}{r}0,004 \\
0,000 \\
0,014 \\
-0,382 \\
0,026 \\
\end{array}$ & $\begin{array}{l}0,508 \\
0,007 \\
0,672 \\
0,000 \\
0,420 \\
\end{array}$ & $\begin{array}{r}-0,010 \\
0,000 \\
0,137 \\
-0,372 \\
0,015 \\
\end{array}$ & $\begin{array}{l}0,171 \\
0,731 \\
0,000 \\
0,000 \\
0,699 \\
\end{array}$ \\
\hline De asalariados registrados a & & & & \\
\hline $\begin{array}{l}\text { Tamaño empresa: }>500 \\
\text { Tamaño empresa: } 101-500 \\
\text { Tamaño empresa: } 26-100 \\
\text { Tamaño empresa: } 6-25 \\
\text { Edad } \\
\text { Edad al cuadrado } \\
\text { Sexo masculino } \\
\text { Nivel bajo de educación } \\
\text { Jefe de familia }\end{array}$ & $\begin{array}{r}-0,888 \\
-0,799 \\
-0,683 \\
-0,412 \\
-0,073 \\
0,001 \\
0,242 \\
0,182 \\
-0,133 \\
\end{array}$ & $\begin{array}{l}0,000 \\
0,000 \\
0,000 \\
0,000 \\
0,000 \\
0,000 \\
0,000 \\
0,000 \\
0,001 \\
\end{array}$ & $\begin{array}{r}-1,155 \\
-0,954 \\
-0,837 \\
-0,544 \\
-0,063 \\
0,001 \\
0,156 \\
-0,234 \\
-0,102 \\
\end{array}$ & $\begin{array}{l}0,000 \\
0,000 \\
0,000 \\
0,000 \\
0,000 \\
0,001 \\
0,009 \\
0,000 \\
0,089 \\
\end{array}$ \\
\hline De asalariados no registrado & & & & \\
\hline $\begin{array}{l}\text { Tamaño empresa: }>500 \\
\text { Tamaño empresa: } 101-500 \\
\text { Tamaño empresa: } 26-100 \\
\text { Tamaño empresa: } 6-25 \\
\text { Edad } \\
\text { Edad al cuadrado } \\
\text { Sexo masculino } \\
\text { Nivel bajo de educación } \\
\text { Jefe de familia }\end{array}$ & $\begin{array}{r}1,148 \\
1,508 \\
1,282 \\
0,698 \\
0,006 \\
0,000 \\
-0,106 \\
-0,305 \\
0,083\end{array}$ & $\begin{array}{l}0,000 \\
0,000 \\
0,000 \\
0,000 \\
0,506 \\
0,484 \\
0,031 \\
0,000 \\
0,096\end{array}$ & $\begin{array}{r}0,797 \\
1,535 \\
1,333 \\
0,756 \\
0,035 \\
0,000 \\
-0,141 \\
0,318 \\
-0,073\end{array}$ & $\begin{array}{l}0,000 \\
0,000 \\
0,000 \\
0,000 \\
0,003 \\
0,002 \\
0,014 \\
0,000 \\
0,189\end{array}$ \\
\hline
\end{tabular}

De asalariados registrados a asalariados no registrados

(excluido el tamaño de la empresa como variable independiente)

\begin{tabular}{|c|c|c|c|c|}
\hline Edad & $-0,080$ & 0,000 & $-0,066$ & 0,000 \\
\hline Edad al cuadrado & 0,001 & 0,000 & 0,001 & 0,000 \\
\hline Sexo masculino & 0,046 & 0,225 & 0,071 & 0,167 \\
\hline Nivel bajo de educación & 0,398 & 0,000 & 0,385 & 0,000 \\
\hline Jefe de familia & $-0,148$ & 0,000 & $-0,111$ & 0,033 \\
\hline \multicolumn{5}{|c|}{$\begin{array}{l}\text { De asalariados no registrados a asalariados registrados } \\
\text { (excluido el tamaño de la empresa como variable independiente) }\end{array}$} \\
\hline Edad & 0,025 & 0,001 & 0,032 & 0,000 \\
\hline Edad al cuadrado & 0,000 & 0,002 & 0,000 & 0,000 \\
\hline Sexo masculino & 0,370 & 0,000 & 0,354 & 0,000 \\
\hline Nivel bajo de educación & $-0,594$ & 0,000 & $-0,629$ & 0,000 \\
\hline Jefe de familia & 0,157 & 0,000 & $-0,037$ & 0,424 \\
\hline
\end{tabular}

Fuente: Elaboración propia sobre la base de datos de las encuestas permanentes de hogares.

Nota: Sig: Significación. 
CUADRO 5

Argentina: diferencias de los ingresos por hora según tipo de empleo ${ }^{a}$, 1996-2003 y 2004-2006

(Todas las áreas urbanas encuestadas)

\begin{tabular}{|c|c|c|c|c|c|c|c|c|c|}
\hline \multirow{2}{*}{$\begin{array}{l}\text { Empleo } \\
\text { destino }\end{array}$} & \multirow{2}{*}{$\begin{array}{l}\text { Empleo } \\
\text { origen }\end{array}$} & \multicolumn{4}{|c|}{ 1996-2003 } & \multicolumn{4}{|c|}{ 2004-2006 } \\
\hline & & $\begin{array}{c}\text { Tratamiento } \\
\mathrm{N}\end{array}$ & $\begin{array}{c}\text { Control } \\
\mathrm{N}\end{array}$ & $\begin{array}{c}\text { Diferencia } \\
\text { de ingresos }\end{array}$ & $t$ & $\begin{array}{c}\text { Tratamiento } \\
\mathrm{N}\end{array}$ & $\begin{array}{c}\text { Control } \\
\mathrm{N}\end{array}$ & $\begin{array}{c}\text { Diferencia } \\
\text { de ingresos }\end{array}$ & $t$ \\
\hline \multicolumn{10}{|l|}{ Panel 1} \\
\hline $\mathrm{EI}^{\mathrm{b}}$ & $\mathrm{EF}^{\mathrm{c}}$ & 1859 & 18089 & $-0,139$ & $-8,544$ & 800 & 10556 & $-0,169$ & $-6,846$ \\
\hline $\mathrm{EF}$ & EI & 2226 & 15632 & 0,127 & 8,408 & 1511 & 9579 & 0,193 & 10,231 \\
\hline ESI $^{\mathrm{d}}$ & $\mathrm{ESF}^{\mathrm{e}}$ & 2932 & 16906 & $-0,078$ & $-6,215$ & 1361 & 11725 & $-0,092$ & $-4,933$ \\
\hline ESF & ESI & 3095 & 12091 & 0,045 & 3,162 & 1791 & 6771 & 0,107 & 5,633 \\
\hline \multicolumn{10}{|l|}{ Panel 2} \\
\hline $\operatorname{AIR}^{\mathrm{f}}$ & $\mathrm{AFR}^{\mathrm{g}}$ & 1087 & 14308 & $-0,008$ & $-0,633$ & 341 & 7608 & 0,004 & 0,199 \\
\hline AFR & AIR & 1135 & 1829 & 0,008 & 0,404 & 443 & 649 & 0,034 & 1,147 \\
\hline $\mathrm{AINR}^{\mathrm{h}}$ & $\mathrm{AFNR}^{\mathrm{i}}$ & 480 & 1649 & $-0,061$ & $-1,588$ & 392 & 1446 & $-0,091$ & $-2,317$ \\
\hline AFNR & AINR & 548 & 2567 & $-0,050$ & $-1,488$ & 429 & 1633 & $-0,014$ & $-0,374$ \\
\hline \multicolumn{10}{|l|}{ Panel 3} \\
\hline AFNR & AFR & 511 & 12662 & $-0,066$ & $-2,487$ & 270 & 6495 & $-0,163$ & $-4,89$ \\
\hline AFR & AFNR & 707 & 1685 & 0,076 & 2,622 & 579 & 1434 & 0,185 & 5,733 \\
\hline AINR & AIR & 213 & 1641 & $-0,118$ & $-2,904$ & 79 & 382 & $-0,116$ & $-1,557$ \\
\hline AIR & AINR & 258 & 2359 & 0,017 & 0,424 & 156 & 1176 & 0,083 & 1,603 \\
\hline
\end{tabular}

Panel 4

4.1 Excluido el tamaño como variable independiente

\begin{tabular}{|c|c|c|c|c|c|c|c|c|c|}
\hline $\mathrm{ANR}^{\mathrm{j}}$ & $\mathrm{AR}^{\mathrm{k}}$ & 1078 & 16462 & $-0,119$ & $-6,246$ & 481 & 8431 & $-0,194$ & $-7,138$ \\
\hline $\mathrm{AR}$ & ANR & 1347 & 4508 & 0,095 & 4,775 & 1000 & 3435 & 0,203 & 8,711 \\
\hline \multicolumn{10}{|c|}{ 4.2 Incluido el tamaño como variable independiente } \\
\hline ANR & $\mathrm{AR}$ & 1078 & 10019 & $-0,109$ & $-5,544$ & 429 & 3176 & $-0,197$ & $-6,273$ \\
\hline $\mathrm{AR}$ & ANR & 1347 & 3804 & 0,099 & 3,665 & 901 & 2172 & 0,138 & 4,061 \\
\hline \multicolumn{10}{|c|}{ Panel 5} \\
\hline TINA $^{1}$ & AFR & 380 & 10995 & $-0,184$ & $-4,285$ & 124 & 4067 & $-0,244$ & $-3,384$ \\
\hline AFR & TINA & 306 & 6220 & 0,212 & 4,310 & 172 & 2515 & 0,425 & 6,769 \\
\hline TINA & AFNR & 349 & 1542 & $-0,155$ & $-3,203$ & 232 & 1231 & $-0,067$ & $-1,06$ \\
\hline AFNR & TINA & 363 & 5639 & $-0,014$ & $-0,296$ & 283 & 2952 & $-0,05$ & $-0,888$ \\
\hline TINA & AIR & 150 & 1442 & $-0,029$ & $-0,550$ & 47 & 221 & 0,053 & 0,555 \\
\hline AIR & TINA & 125 & 4261 & 0,000 & 0,004 & 35 & 784 & $-0,083$ & $-0,731$ \\
\hline TINA & AINR & 793 & 2441 & $-0,004$ & $-0,131$ & 489 & 1728 & 0,001 & 0,026 \\
\hline AINR & TINA & 766 & 6924 & $-0,117$ & $-3,375$ & 477 & 3351 & $-0,002$ & $-0,058$ \\
\hline
\end{tabular}

Fuente: Elaboración propia sobre la base de datos de las encuestas permanentes de hogares.

Nota: N: Cantidad; $t$ : Tiempo.

a Excluidos los empleados domésticos y los beneficiarios de programas de empleo.

b EI: Empleo informal.

c EF: Empleo formal.

d ESI: Empleo en el sector informal.

e ESF: Empleo en el sector formal.

f AIR: Asalariado informal registrado.

$\mathrm{g}$ AFR: Asalariado formal registrado.

h AINR: Asalariado informal no registrado.

i AFNR: Asalariado formal no registrado.

j ANR: Asalariado no registrado.

k RWE AR: Asalariado registrado.

1 TINA: Trabajador informal no asalariado. 
Las diferencias salariales entre el empleo formal y el informal son mayores que entre el empleo en el sector formal y el sector informal, lo que sugiere que la causa principal de estas diferencias podría ser la condición jurídica de los asalariados. Las grandes diferencias que resultan al comparar los ingresos de los empleados registrados y los no registrados parece confirmar lo anterior.

Con el fin de medir la repercusión del tamaño de la empresa y el tipo de contrato laboral en la brecha de ingresos, se repitió el ejercicio tomando otros grupos de trabajadores más restringidos. Dado que las empresas más grandes suelen tener una mayor proporción de empleados registrados, la prima salarial del empleo formal parece más bien relacionarse con el tamaño. Sin embargo, si se comparan los ingresos dentro del grupo de los asalariados registrados o de los no registrados exclusivamente, la diferencia de ingresos entre los trabajadores del sector informal y el sector formal se acerca a 0 si se evalúa según el tipo de contrato laboral (véase el cuadro 5, panel 2). Únicamente se observó una gran pérdida de ingresos en el caso de los asalariados no registrados que pasaban de una gran empresa a otra más pequeña durante el segundo período. Cabe notar que esta pérdida equivale a aproximadamente la mitad de la prima de registro.

Al comparar los ingresos de empleados con diferentes contratos laborales según el tamaño de la empresa, si se mide la diferencia entre el sector formal y el informal se observa una diferencia notable con respecto a 0 en la mayoría de los ejercicios (véase el cuadro 5, panel 3). Los resultados no varían al utilizar un método alternativo (comparación por puntajes estimados que incluyen variables independientes) para evaluar el impacto del tamaño de la empresa. Los promedios de las diferencias de ingresos entre asalariados registrados y no registrados son similares (véase el cuadro 5, paneles 1 y 4). Esto reafirma la hipótesis de que existe una amplia segmentación basada en la condición jurídica del trabajador, sea cual sea el tamaño de la empresa en que trabaja.

En cuanto a la categoría laboral, no existen diferencias si se compara a los trabajadores no asalariados con los empleados de pequeñas empresas, independientemente del tipo de contrato laboral (excepto en uno de los casos). Sin embargo, los asalariados registrados tienden a perder ingresos relativos cuando se convierten en trabajadores autónomos, y las ganancias de los trabajadores no asalariados que asumen un puesto formal, en contrapartida, aumentan más que si hubieran continuado en la informalidad. De este modo, estos ejercicios sugieren que los trabajadores no asalariados del sector informal ganan menos que los empleados del sector formal.

Tras los resultados mencionados, que constituyen promedios para todos los tipos de trabajadores, podrían ocultarse elementos heterogéneos: ciertos grupos pueden tener diferentes probabilidades de transición o percibir diferentes ingresos. No obstante, las brechas de ingresos estimadas para los distintos subgrupos de trabajadores parecen mostrar que sigue existiendo segmentación en los diferentes grupos identificados, como se puede observar en el cuadro 6. Incluso si se tiene en cuenta la edad, la educación, el sexo y las diferentes probabilidades de transición, estimadas sobre la base de los puntajes del grupo, siempre existe una penalización en el ingreso. $\mathrm{Si}$ bien puede no ser una gran penalización, los ingresos de los hombres y de los trabajadores con un bajo nivel de educación experimentan mayores pérdidas cuando adoptan la informalidad, y mayores ganancias cuando la abandonan.

Argentina: diferencias de los ingresos por hora, 1996-2003 y 2004-2006

(Todas las áreas urbanas encuestadas)

\begin{tabular}{|c|c|c|c|c|}
\hline & \multicolumn{2}{|l|}{$1996-2003$} & \multicolumn{2}{|l|}{ 2004-2006 } \\
\hline & Diferencia de ingresos & $t$ & Diferencia de ingresos & $t$ \\
\hline \multicolumn{5}{|c|}{ Transición al empleo informal } \\
\hline $\begin{array}{l}\text { Puntajes Q } 1 \\
\text { Puntajes Q } 2 \\
\text { Puntajes Q } 3 \\
\text { Puntajes Q } 4 \\
\text { Sexo femenino } \\
\text { Sexo masculino } \\
\text { Nivel bajo de educación } \\
\text { Nivel alto de educación } \\
\text { Edad }<30 \\
\text { Edad }>30\end{array}$ & $\begin{array}{l}-0,088 \\
-0,095 \\
-0,148 \\
-0,168 \\
-0,119 \\
-0,146 \\
-0,174 \\
-0,092 \\
-0,142 \\
-0,137\end{array}$ & $\begin{array}{l}-2,111 \\
-2,448 \\
-4,739 \\
-6,608 \\
-3,925 \\
-7,581 \\
-7,886 \\
-3,861 \\
-5,295 \\
-6,709\end{array}$ & $\begin{array}{l}-0,209 \\
-0,030 \\
-0,139 \\
-0,239 \\
-0,088 \\
-0,203 \\
-0,227 \\
-0,123 \\
-0,171 \\
-0,168\end{array}$ & $\begin{array}{l}-2,782 \\
-0,508 \\
-2,866 \\
-6,765 \\
-1,778 \\
-7,251 \\
-6,058 \\
-3,799 \\
-4,097 \\
-5,515\end{array}$ \\
\hline
\end{tabular}


Cuadro 6 (conclusión)

\begin{tabular}{|c|c|c|c|c|}
\hline & \multicolumn{2}{|l|}{$1996-2003$} & \multicolumn{2}{|l|}{ 2004-2006 } \\
\hline & Diferencia de ingresos & $t$ & Diferencia de ingresos & $t$ \\
\hline \multicolumn{5}{|c|}{ Transición al empleo formal } \\
\hline $\begin{array}{l}\text { Puntajes Q } 1 \\
\text { Puntajes Q } 2 \\
\text { Puntajes Q } 3 \\
\text { Puntajes Q } 4 \\
\text { Sexo femenino } \\
\text { Sexo masculino } \\
\text { Nivel bajo de educación } \\
\text { Nivel alto de educación } \\
\text { Edad <30 } \\
\text { Edad >30 }\end{array}$ & $\begin{array}{l}0,048 \\
0,114 \\
0,109 \\
0,174 \\
0,112 \\
0,136 \\
0,090 \\
0,179 \\
0,132 \\
0,124 \\
\end{array}$ & $\begin{array}{l}1,375 \\
3,382 \\
3,374 \\
7,486 \\
4,617 \\
7,023 \\
4,630 \\
7,658 \\
5,640 \\
6,284 \\
\end{array}$ & $\begin{array}{l}0,132 \\
0,188 \\
0,193 \\
0,212 \\
0,143 \\
0,224 \\
0,214 \\
0,175 \\
0,260 \\
0,141 \\
\end{array}$ & $\begin{array}{l}2,942 \\
4,489 \\
4,988 \\
7,284 \\
4,949 \\
9,094 \\
8,369 \\
6,596 \\
9,208 \\
5,620 \\
\end{array}$ \\
\hline \multicolumn{5}{|c|}{ Transición al empleo en el sector informal } \\
\hline $\begin{array}{l}\text { Puntajes Q } 1 \\
\text { Puntajes Q } 2 \\
\text { Puntajes Q } 3 \\
\text { Puntajes Q } 4 \\
\text { Sexo femenino } \\
\text { Sexo masculino } \\
\text { Nivel bajo de educación } \\
\text { Nivel alto de educación } \\
\text { Edad <30 } \\
\text { Edad }>30\end{array}$ & $\begin{array}{l}-0,068 \\
-0,043 \\
-0,087 \\
-0,096 \\
-0,077 \\
-0,079 \\
-0,098 \\
-0,052 \\
-0,083 \\
-0,076 \\
\end{array}$ & $\begin{array}{l}-2,389 \\
-1,664 \\
-3,691 \\
-4,218 \\
-3,519 \\
-5,162 \\
-5,520 \\
-3,002 \\
-3,727 \\
-4,982 \\
\end{array}$ & $\begin{array}{l}-0,038 \\
-0,061 \\
-0,075 \\
-0,131 \\
-0,036 \\
-0,112 \\
-0,107 \\
-0,077 \\
-0,111 \\
-0,082\end{array}$ & $\begin{array}{l}-0,762 \\
-1,389 \\
-2,050 \\
-4,538 \\
-0,981 \\
-5,156 \\
-3,898 \\
-3,056 \\
-3,561 \\
-3,504 \\
\end{array}$ \\
\hline \multicolumn{5}{|c|}{ Transición al empleo en el sector formal } \\
\hline $\begin{array}{l}\text { Puntajes Q } 1 \\
\text { Puntajes Q } 2 \\
\text { Puntajes Q } 3 \\
\text { Puntajes Q } 4 \\
\text { Sexo femenino } \\
\text { Sexo masculino } \\
\text { Nivel bajo de educación } \\
\text { Nivel alto de educación } \\
\text { Edad <30 } \\
\text { Edad >30 }\end{array}$ & $\begin{array}{r}-0,015 \\
0,068 \\
0,039 \\
0,058 \\
0,052 \\
0,043 \\
0,043 \\
0,045 \\
0,057 \\
0,038 \\
\end{array}$ & $\begin{array}{r}-0,432 \\
2,325 \\
1,336 \\
2,667 \\
1,891 \\
2,589 \\
2,300 \\
2,185 \\
2,298 \\
2,212 \\
\end{array}$ & $\begin{array}{l}0,077 \\
0,078 \\
0,100 \\
0,142 \\
0,074 \\
0,118 \\
0,118 \\
0,096 \\
0,147 \\
0,082 \\
\end{array}$ & $\begin{array}{l}1,513 \\
1,977 \\
2,817 \\
4,533 \\
1,952 \\
5,377 \\
4,315 \\
3,720 \\
4,689 \\
3,458 \\
\end{array}$ \\
\hline \multicolumn{5}{|c|}{ Transición a asalariado no registrado } \\
\hline $\begin{array}{l}\text { Puntajes Q } 1 \\
\text { Puntajes Q } 2 \\
\text { Puntajes Q } 3 \\
\text { Puntajes Q } 4 \\
\text { Sexo femenino } \\
\text { Sexo masculino } \\
\text { Nivel bajo de educación } \\
\text { Nivel alto de educación } \\
\text { Edad <30 } \\
\text { Edad >30 }\end{array}$ & $\begin{array}{l}-0,101 \\
-0,132 \\
-0,081 \\
-0,138 \\
-0,086 \\
-0,131 \\
-0,149 \\
-0,079 \\
-0,113 \\
-0,123 \\
\end{array}$ & $\begin{array}{l}-1,789 \\
-2,874 \\
-2,161 \\
-4,941 \\
-2,673 \\
-5,645 \\
-5,635 \\
-2,946 \\
-3,976 \\
-4,835 \\
\end{array}$ & $\begin{array}{l}-0,245 \\
-0,020 \\
-0,221 \\
-0,227 \\
-0,071 \\
-0,243 \\
-0,247 \\
-0,151 \\
-0,200 \\
-0,189\end{array}$ & $\begin{array}{l}-3,373 \\
-0,287 \\
-4,068 \\
-5,827 \\
-1,436 \\
-7,577 \\
-5,983 \\
-4,220 \\
-4,607 \\
-5,451 \\
\end{array}$ \\
\hline \multicolumn{5}{|c|}{ Transición a asalariado registrado } \\
\hline $\begin{array}{l}\text { Puntajes Q } 1 \\
\text { Puntajes Q } 2 \\
\text { Puntajes Q } 3 \\
\text { Puntajes Q } 4 \\
\text { Sexo femenino } \\
\text { Sexo masculino } \\
\text { Nivel bajo de educación } \\
\text { Nivel alto de educación } \\
\text { Edad <30 } \\
\text { Edad }>30\end{array}$ & $\begin{array}{l}0,092 \\
0,044 \\
0,104 \\
0,119 \\
0,106 \\
0,089 \\
0,081 \\
0,111 \\
0,116 \\
0,072\end{array}$ & $\begin{array}{l}1,841 \\
1,044 \\
2,962 \\
3,544 \\
3,050 \\
3,787 \\
3,102 \\
3,795 \\
4,350 \\
2,460\end{array}$ & $\begin{array}{l}0,249 \\
0,140 \\
0,245 \\
0,177 \\
0,174 \\
0,208 \\
0,190 \\
0,213 \\
0,235 \\
0,156\end{array}$ & $\begin{array}{l}4,592 \\
2,782 \\
5,691 \\
4,341 \\
3,962 \\
7,602 \\
5,619 \\
6,763 \\
7,498 \\
4,525\end{array}$ \\
\hline
\end{tabular}

Fuente: Elaboración propia sobre la base de datos de las encuestas permanentes de hogares.

Nota: $t$ : Tiempo; Q: Grupo de control. 


\section{VI}

\section{Conclusiones}

Dadas la magnitud y persistencia del mercado laboral informal en la Argentina, la existencia de diferencias en los ingresos entre la formalidad y la informalidad es una cuestión de especial relevancia. En este artículo se ha explorado esta cuestión a partir de dos definiciones diferentes de informalidad: la interpretación convencional, de acuerdo con la perspectiva de la OIT, y una segunda definición que tiene en cuenta el cumplimiento de los aspectos legales y regulatorios. Según la primera, la unidad informal suele operar en actividades de fácil inserción y presenta una división poco clara entre el capital y la mano de obra (empleo en el sector informal). La informalidad también puede definirse como la falta de cumplimiento de la normativa laboral $\mathrm{u}$ otras por parte de ciertas empresas (empleo informal). En 2005, el empleo informal constituyó el 55\% del empleo urbano en la Argentina, mientras que el empleo en el sector informal representó el 38\% del total.

Los datos empíricos sobre la segmentación basados en diferenciales de ingresos son cuestionables debido a los métodos paramétricos utilizados. Para contrarrestar este hecho, se han utilizado técnicas semiparamétricas. El cotejo de los puntajes de propensión, aplicado a los datos de panel, permite comparar la variación de los ingresos de los trabajadores formales que permanecen en este mismo sector con las diferencias que experimentan los trabajadores que transitan del sector formal al informal, y viceversa. La ausencia de diferencia es un claro indicio de que no existe segmentación. Las comparaciones se realizan entre trabajadores con una propensión similar a la transición sectorial, sobre la base de características observables. Se estudian dos subperíodos diferentes: el comprendido entre 1996 y 2003, cuando se produjo un repunte en el número de asalariados informales, y el lapso 2004-2006, en que el sector informal permaneció sin grandes cambios. Ambos períodos coinciden también con el mencionado cambio en el método de recolección de datos para la Encuesta Permanente de Hogares.

Los resultados reafirman la hipótesis de que el mercado laboral urbano de la Argentina se encuentra segmentado. Trabajadores que presentan una propensión similar para acceder a un determinado sector o salir de él, según características observables, perciben ingresos diferentes. Las diferencias en los ingresos son mayores entre el empleo formal y el informal que entre el empleo del sector formal y el del sector informal, lo que hace pensar que la condición jurídica del empleado es la causa principal de las diferencias. Las mayores disparidades que se observan al comparar los ingresos de los trabajadores registrados y los no registrados corroboran esta impresión, y evidencian que la segmentación basada en la condición jurídica de los trabajadores es una realidad generalizada. Si se comparan las brechas de ingresos de los diferentes subgrupos de trabajadores se puede observar la misma tendencia, lo que confirma que los trabajadores no asalariados del sector informal ganan menos que los empleados en el sector formal.

\section{Bibliografía}

Beccaria, L. y F. Groisman (2008), "Informalidad y pobreza en Argentina”, Investigación Económica, vol. 67, № 266, Ciudad de México.

De Soto, H. (1987), El otro sendero, Buenos Aires, Editorial Sudamericana.

Dickens, W. y K. Lang (1988), "The reemergence of segmented labor market theory", American Economic Review, vol. 78, $\mathrm{N}^{\circ}$ 2, Nashville, Tennessee, American Economic Association.

Evans, D.C. y L.S. Leighton (1989), "Some empirical aspects of entrepreneurship", American Economic Review, vol. 79, $\mathrm{N}^{\circ} 3$, Nashville, Tennessee, American Economic Association.

Fields, G. (1990), "Labour market modelling and the urban informal sector: theory and evidence", The Informal Sector Revisited, D. Turnham, B. Salomé y A. Schwarz (comps.), París, Organización para la Cooperación y el Desarrollo Económicos (OCDE).

(1975), "Rural-urban migration, urban unemployment and under-development and job-search security in LCDs", Journal of Development Economics, vol. 2, Nㅜ 2, Amsterdam, Elsevier.
Harris, J.R. y M.P. Todaro (1970), "Migration, unemployment and development: a two-sector analysis", American Economic Review, vol. $60, \mathrm{~N}^{\circ} 1$, Nashville, Tennessee, American Economic Association.

Hart, K. (1973), "Informal income opportunities and urban employment in Ghana", The Journal of Modern African Studies, vol. 11, $\mathrm{N}^{\circ}$ 1, Cambridge, Cambridge University Press.

Heckman, J. y V. Hotz (1986), "An investigation of labor market earnings of Panamanian males", Journal of Human Resources, vol. 21, $\mathrm{N}^{\circ} 4$, Wisconsin, University of Wisconsin Press.

Heckman, J. y G. Sedlacek (1985), "Heterogeneity, aggregation, and market wages functions: an empirical model of self-selection in the labor market", Journal of Political Economy, vol. 93, $\mathrm{N}^{\circ} 6$, Chicago, The University of Chicago Press.

Hussmanns, R. (2005), "Measuring the informal economy: from employment in the informal sector to informal employment", Working Paper, $\mathrm{N}^{\circ}$ 53, Ginebra, Organización Internacional del Trabajo (OIT). 
Killingsworth, M. (1987), "Heterogeneous preferences, compensating wage differentials, and comparable worth", The Quarterly Journal of Economics, vol. 102, $\mathrm{N}^{\circ} 4$, Cambridge, Massachusetts, The MIT Press, noviembre.

Lewis, A. (1954), "Economic development with unlimited supplies of labour", The Manchester School, vol. 22, $\mathrm{N}^{\circ}$ 2, Wiley.

Magnac, T. (1991), "Segmented or competitive labour markets", Econometrica, vol. 59, $\mathrm{N}^{\circ} 1$

Maloney, W.F. (1999), "Does informality imply segmentation in urban labor markets? Evidence from sectoral transitions in Mexico", World Bank Economic Review, vol. 13, N 2, Washington, D.C., Banco Mundial.

oIT (Organización Internacional del Trabajo) (1972), Employment, Incomes and Equality: A Strategy for Increasing Productive Employment in Kenya, Ginebra.

Packard, T. (2007), "Do workers in Chile choose informal employment? A dynamic analysis of sector choice", Policy Research Working Paper, $\mathrm{N}^{\circ}$ 4232, Washington, D.C., Banco Mundial.
Pagés-Serra, C. y M. Stampini (2007), "No education, no good jobs? Evidence on the relationship between education and labor market segmentation", Research Department Working Paper Series, $\mathrm{N}^{\circ}$ 627, Washington, D.C., Banco Interamericano de Desarrollo.

Perry, G. y otros (2007), Informality: Exit and Exclusion in Latin America, Washington, D.C., Banco Mundial.

Portes, A., M. Castells y L.A. Benton (1989), The Informal Economy: Studies in Advanced and Less Developed Countries, Baltimore, Johns Hopkins University Press.

Pradhan, M. y A. van Soest (1995), "Formal and informal sector employment in urban areas of Bolivia", Labour Economics, vol. 2, $\mathrm{N}^{\circ}$ 3, Amsterdam, Elsevier.

Pratap, S. y E. Quintín (2006), "Are labour markets segmented in developing countries? A semiparametric approach", European Economic Review, vol. 50, $\mathrm{N}^{\circ} 7$, SAGE.

Sánchez, C., H. Palmieri y F. Ferrero (1976), "Desarrollo industrial urbano y empleo en la ciudad de Córdoba (Argentina): un caso de crecimiento desequilibrado", Ginebra, Organización Internacional del Trabajo (OIT), inédito. 